\title{
Microstructure and Mechanical Properties of X80 Pipeline Steel Joints by Friction Stir Welding Under Various Cooling Conditions
}

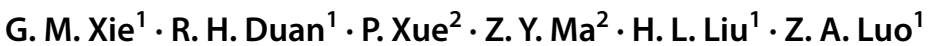 \\ Received: 14 April 2019 / Revised: 27 May 2019 / Published online: 23 July 2019 \\ (c) The Chinese Society for Metals (CSM) and Springer-Verlag GmbH Germany, part of Springer Nature 2019
}

\begin{abstract}
X80 pipeline steel plates were friction stir welded (FSW) under air, water, liquid $\mathrm{CO}_{2}+$ water, and liquid $\mathrm{CO}_{2}$ cooling conditions, producing defect-free welds. The microstructural evolution and mechanical properties of these FSW joints were studied. Coarse granular bainite was observed in the nugget zone (NZ) under air cooling, and lath bainite and lath martensite increased significantly as the cooling medium temperature reduced. In particular, under the liquid $\mathrm{CO}_{2}$ cooling condition, a dual phase structure of lath martensite and fine ferrite appeared in the NZ. Compared to the case under air cooling, a strong shear texture was identified in the NZs under other rapid cooling conditions, because the partial deformation at elevated temperature was retained through higher cooling rates. Under liquid $\mathrm{CO}_{2}$ cooling, the highest transverse tensile strength and elongation of the joint reached $92 \%$ and $82 \%$ of those of the basal metal (BM), respectively, due to the weak tempering softening. A maximum impact energy of up to $93 \%$ of that of the $\mathrm{BM}$ was obtained in the $\mathrm{NZ}$ under liquid $\mathrm{CO}_{2}$ cooling, which was attributed to the operation of the dual phase of lath martensite and fine ferrite.
\end{abstract}

Keywords Friction stir welding $\cdot$ Pipeline steel $\cdot$ Microstructure $\cdot$ Mechanical properties $\cdot$ Cooling

\section{Introduction}

With the rapid development of the economy, the demand for energy has increased remarkably. Presently, oil and natural gas are mainly transported by means of pipelines. Therefore, pipeline steels with both high strength and excellent toughness are being developed to enhance the transport efficiency [1-3]. The welding process plays an essential role for laying pipelines, and fusion welding techniques such as submerged arc welding and gas metal arc welding (GMAW) are widely used by virtue of their advantages of high efficiency and low cost $[4,5]$.

It should be pointed out that the fairly high heat input for fusion welding easily results in the formation of coarse

Available online at http://link.springer.com/journal/40195

G. M. Xie

xiegm@ral.neu.edu.cn

1 State Key Laboratory of Rolling and Automation, Northeastern University, Shenyang 110819, China

2 Shenyang National Laboratory for Materials Science, Institute of Metal Research, Chinese Academy of Sciences, Shenyang 110016, China grains and large M-A islands in the vicinity of the fusion line. These coarse grains are quite susceptible to hydrogeninduced cracking [1,2]. Besides, the coarse M-A constituents distributed along the grain boundaries can cause an intense stress concentration during deformation and then become crack sources, eventually leading to crack initiation and propagation [6]. Moreover, in fusion welded joints, there are usually some solidification defects, including voids and cracks. Thus, the tensile properties and impact toughness of fusion welded joints are significantly deteriorated $[1,7]$.

During operation of oil and gas pipelines, the welded joints often suffer from high pressures and low environmental temperatures. As a result, the welded joints are required to have an excellent toughness in order to ensure the security of oil and gas pipelines. However, it is difficult to avoid the coarse-grained heat-affected zone (CGHAZ) near the fusion line $[8,9]$. Therefore, it is urgent to develop a new welding technique to obtain the high-quality pipeline joints necessary for oil and gas transportation.

Friction stir welding (FSW) is a new solid-state joining technique. Fine and equiaxed recrystallized grains are produced in the nugget zone (NZ) by the severe plastic deformation and frictional heat generated through the non-consumable rotating tool [10]. In comparison with the case of 
fusion welding, no remarkable coarse grains are detected in the heat-affected zone (HAZ) and no defects such as cracks, voids, and segregation are found in the NZ because of the solid-state nature of FSW, thereby resulting in an improvement in the mechanical properties of FSW joints $[11,12]$. In addition, FSW is an automatic welding process, and no filler metal and flux are used. Therefore, FSW is a desirable and suitable technique for joining pipeline steels.

In the past few years, several studies on FSW pipeline steels revealed that high heat input parameters (high rotation rate and/or low traverse speed) are required to ensure a sufficient metal flow of the pipeline steels at elevated temperatures during FSW [13-16]. Relative to the CGHAZ, the recrystallized grains in the NZ are coarsened to a certain extent, although a dynamic recrystallization occurs in the NZ $[15,16]$. Recently, some researchers have suggested that the external rapid cooling during FSW is an effective way to suppress the coarsening of the recrystallized grains by reducing the peak temperature and duration time [17-21]. However, rapid cooling has been primarily used in FSW of $\mathrm{Al}$ or $\mathrm{Cu}$ alloys, and only a few investigators have conducted FSW of high-strength steels under rapid cooling conditions. Actually, a large quantity of oil and gas transportation pipelines operate in some extremely cold regions where the environmental temperature is even below $-30^{\circ} \mathrm{C}$, which would cause an obvious loss in the mechanical properties and security of the pipeline steel joints $[8,9]$. Thus, to obtain high quality of the welded joints, an investigation on the influence of the cooling media with various temperatures on the FSW pipeline steel joints is clearly necessary.

In this study, various cooling conditions such as air, water, liquid $\mathrm{CO}_{2}+$ water, and liquid $\mathrm{CO}_{2}$ were utilized during FSW of X80 pipeline steels. The microstructure and mechanical properties of the welded joints were then studied systematically.

\section{Experimental}

Hot-rolled X80 pipeline steel plates, $6 \mathrm{~mm}$ thick, were friction stir bead welded at a constant rotation speed of $400 \mathrm{rpm}$ with a traverse speed of $100 \mathrm{~mm} / \mathrm{min}$ under various cooling conditions of air, water (water + a certain quantity of ice), liquid $\mathrm{CO}_{2}+$ water (1:1, vol.), and liquid $\mathrm{CO}_{2}$ (Fig. 1a). During FSW, the whole steel plate to be welded was placed under the various cooling media. The initial temperatures of the air, water, liquid $\mathrm{CO}_{2}+$ water, and liquid $\mathrm{CO}_{2}$ media were $19,3,-14$, and $-34{ }^{\circ} \mathrm{C}$, respectively, which were measured by means of a thermometer. The chemical composition and mechanical properties of the X80 pipeline steel are listed in Table 1. A W-25Re (wt\%) welding tool with a shoulder $18 \mathrm{~mm}$ in diameter and a tapered pin $7 \mathrm{~mm}$ in diameter and $5 \mathrm{~mm}$ in length was adopted. The tilt angle for all welds was maintained at $3^{\circ}$. The peak temperatures of the NZs were accurately measured by a MegaStir telemetry system placed near the shoulder (Fig. 1b). The peak temperatures
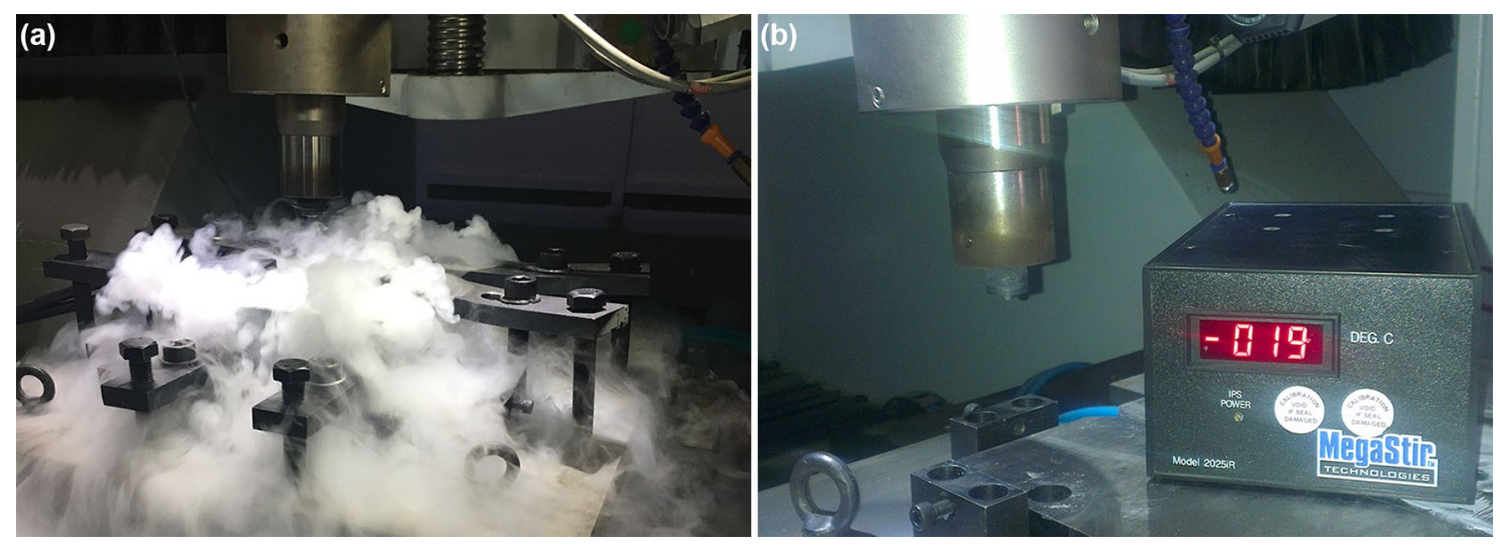

Fig. 1 FSW process under liquid $\mathrm{CO}_{2}$ cooling $\mathbf{a}$, temperature measurement system $\mathbf{b}$

Table 1 Chemical composition and mechanical properties of X80 pipeline steel

\begin{tabular}{|c|c|c|c|c|c|c|c|c|}
\hline Composition (wt\%) & $\mathrm{Fe}$ & $\mathrm{C}$ & $\mathrm{Si}$ & $\mathrm{Mn}$ & $\mathrm{P}$ & $S$ & $\mathrm{Nb}$ & $\mathrm{V}$ \\
\hline & Balance & 0.038 & 0.2329 & 1.611 & 0.0098 & 0.017 & 0.0535 & 0.0029 \\
\hline Mechanical property & \multicolumn{2}{|c|}{ Yield strength (MPa) } & \multicolumn{2}{|c|}{ Tensile strength (MPa) } & \multicolumn{2}{|c|}{ Elongation (\%) } & \multicolumn{2}{|c|}{ Impact energy $\left(\mathrm{J} / \mathrm{cm}^{2}\right)$} \\
\hline & \multicolumn{2}{|l|}{589} & \multicolumn{2}{|l|}{693} & \multicolumn{2}{|l|}{34} & \multicolumn{2}{|l|}{211.6} \\
\hline
\end{tabular}


of the NZs under air, water, liquid $\mathrm{CO}_{2}+$ water, and liquid $\mathrm{CO}_{2}$ cooling conditions reached $1084,981,937$, and $886^{\circ} \mathrm{C}$, respectively.

The microstructural samples were cross-sectioned perpendicular to the FSW direction. The microstructure was characterized using a Leica optical microscope (OM), a Zeiss Ultra-55 field-emission scanning electron microscope (SEM) equipped with an electron back-scatter diffractometer (EBSD), and FEI transmission electron microscope (TEM). The specific location for microstructure analyses is shown by the black frame in Fig. 2a. The samples for the EBSD were electro-polished with a solution of $87 \mathrm{ml}$ ethanol and $13 \mathrm{ml}$ perchloric acid at $25 \mathrm{~V}$ for $25 \mathrm{~s}$. The thin foils for the TEM were prepared as $3 \mathrm{~mm}$ disks and then mechanically ground to $\sim 50 \mu \mathrm{m}$ in thickness, followed by electropolishing at $-20{ }^{\circ} \mathrm{C}$. The $A_{\mathrm{c} 1}$ and $A_{\mathrm{c} 3}$ critical transformation temperatures of the X80 steel measured by a FormastorII phase transformation instrument were 672 and $874{ }^{\circ} \mathrm{C}$, respectively.

Vickers hardness measurements were taken on the crosssectional joints using a $200 \mathrm{~g}$ load and $0.5 \mathrm{~mm}$ spacing by an FM-700 micro-hardness machine. The transverse tensile properties of the welded joints were evaluated at a constant strain rate of $1 \times 10^{-3} \mathrm{~s}^{-1}$ via a SANS tensile machine.
Because the NZ of the FSW micro-alloy steels is typically the lowest toughness zone in the entire joint, only the toughness test of the NZ was performed in this work. The Charpy $\mathrm{V}$-notch impact toughness of the NZs was tested at $20^{\circ} \mathrm{C}$ by an Instron impact machine. The dimensions of the testing samples are shown in Fig. 2b, c.

\section{Results and Discussion}

\subsection{Effect of the Cooling Conditions on the Microstructure of the Joints}

Figure 3 shows macrographs of the FSW joints under different cooling conditions, and basin-like joints without any defects were achieved. The NZ, HAZ, and basal metal (BM) could be clearly distinguished in the FSW X80 pipeline steel joints, and the HAZ was approximately divided into the inside HAZ (IHAZ), middle HAZ (MHAZ), and outside HAZ (OHAZ), which were regarded to experience the various thermal cycle histories. Similarly, Barnes et al. [13] also observed the three subregions in the HAZ of FSW X65 pipeline steel. With the decrease in cooling medium temperature, the HAZ range became narrower as the result

\section{(a) notch location Charpy V-notch specimens}

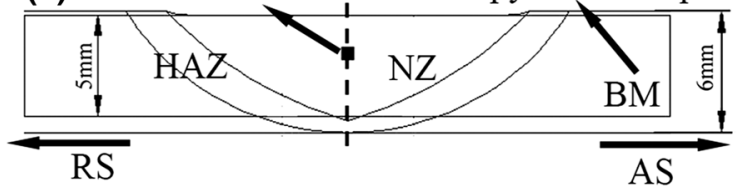

(b)

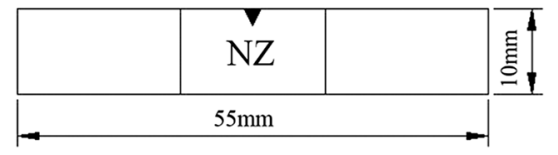

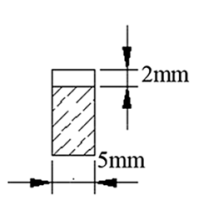

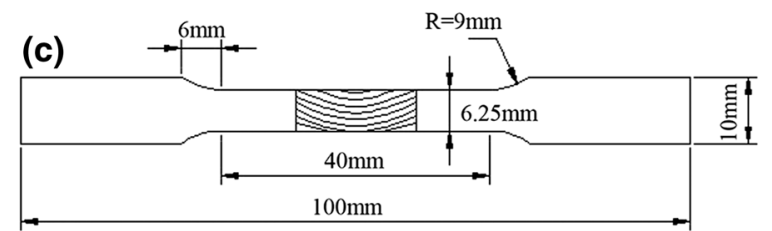

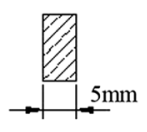

Fig. 2 Schematic diagram of microstructure and property test sample locations a, Charpy V-notch specimen dimension b, tensile specimen dimension $\mathbf{c}$
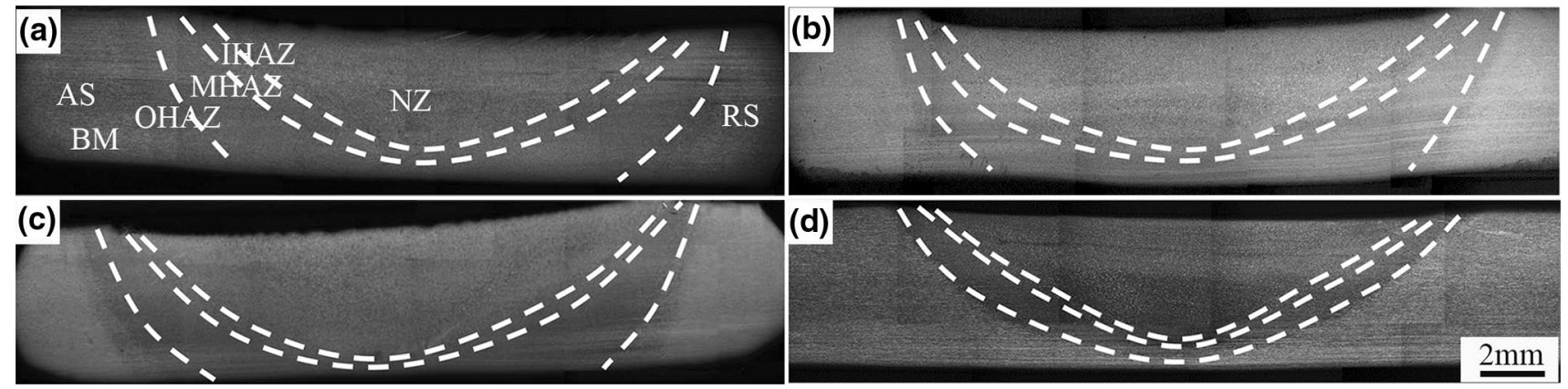

Fig. 3 Macrographs of FSW joints under various cooling conditions: a air cooling, b water cooling, $\mathbf{c}$ liquid $\mathrm{CO}_{2}+$ water cooling, $\mathbf{d}$ liquid $\mathrm{CO}_{2}$ cooling 
of the reduced peak temperature and duration time. Furthermore, for the FSW micro-alloy steel joints, the thermomechanically affected zone (TMAZ) generally could not be discerned due to the existence of the complicated phase transformation.

The microstructural characteristics of the FSW joint obtained under air cooling are shown in Fig. 4. As a typical bainitic steel, the BM was composed of a large amount of fine and intercrossed acicular ferrite with some polygonal ferrite (PF), which contributes to its excellent toughness (Fig. 4a). Compared to the case of the BM, the fine acicular ferrite was transformed to the fine PF in the OHAZ adjacent to the BM (Fig. 4b). However, in the MHAZ, there was a complex phase structure of lath bainite (LB), $\mathrm{PF}$, and granular bainite (GB), while the IHAZ only contained GB and LB (Fig. 4c, d). Clearly, the HAZ exhibited a heterogeneous microstructural distribution, which should be related to the various thermal cycle histories during FSW.

It is widely accepted that the peak temperatures in the OHAZ, MHAZ, and IHAZ were in the temperature ranges of $<A_{\mathrm{cl}}, A_{\mathrm{cl}}-A_{\mathrm{c} 3}$, and $>A_{\mathrm{c} 3}$, respectively [13]. Therefore, in the OHAZ, there were tempering, static recovery, and recrystallization at the peak temperature of $<A_{\mathrm{cl}}$, thereby changing the original acicular ferrite to the fine recrystallized PF in the BM (Fig. 4b). In the MHAZ, the peak temperature was localized just between $A_{\mathrm{c} 1}$ and $A_{\mathrm{c} 3}$. Thus, in the $\mathrm{BM}$ consisting of acicular ferrite and PF, the supersaturated acicular ferrite was preferentially austenitized during FSW and then transformed into LB and GB after FSW, finally achieving a trinal phase structure of $\mathrm{LB}, \mathrm{GB}$, and $\mathrm{PF}$ in the MHAZ (Fig. 4c). The IHAZ close to the NZ experienced a peak temperature higher than $A_{\mathrm{c} 3}$. Therefore, the $\mathrm{BM}$ was fully austenitized during FSW, and then, the coarse austenite was transformed into GB and LB after FSW (Fig. 4d).

Figure $4 \mathrm{e}$ represents the microstructure of the NZ, which comprises the coarse GB and LB. Usually, FSW, as a severe plastic deformation process, can result in the formation of fine recrystallized grains in the NZ [10]. However, in our work, the NZ for the FSW pipeline steel exhibited slightly coarse prior austenitic grains, relative to the IHAZ. Unlike the nonferrous metals, relatively high heat input is required for the FSW steel to ensure the sufficient plastic flow, as the result of the high deformation resistance of steel at elevated temperatures. Similarly, the coarse GB was also found in the NZ of FSW X65 and FSW X100 pipeline steels [13, 16].

Additionally, in this work, a homogeneous microstructure was achieved throughout the NZ from the advancing side to the retreating side. However, many investigations have noted that the NZs of FSW X65 and FSW X80 pipeline steels had a heterogeneous microstructure, where the advancing side contained more low-temperature transformed products, such as lath bainite and lath martensite [11-14]. Actually, this accounts for the fact that the B element from the polycrystalline cubic boron nitride (PCBN) tool inhibited the formation
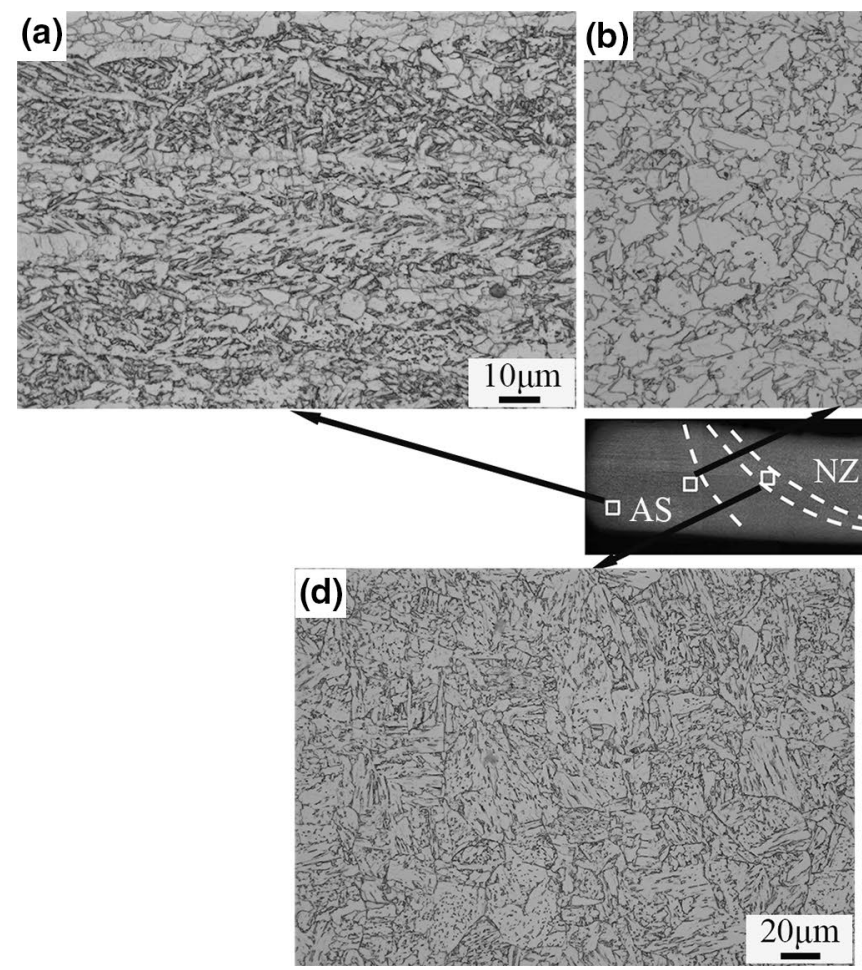

(b)
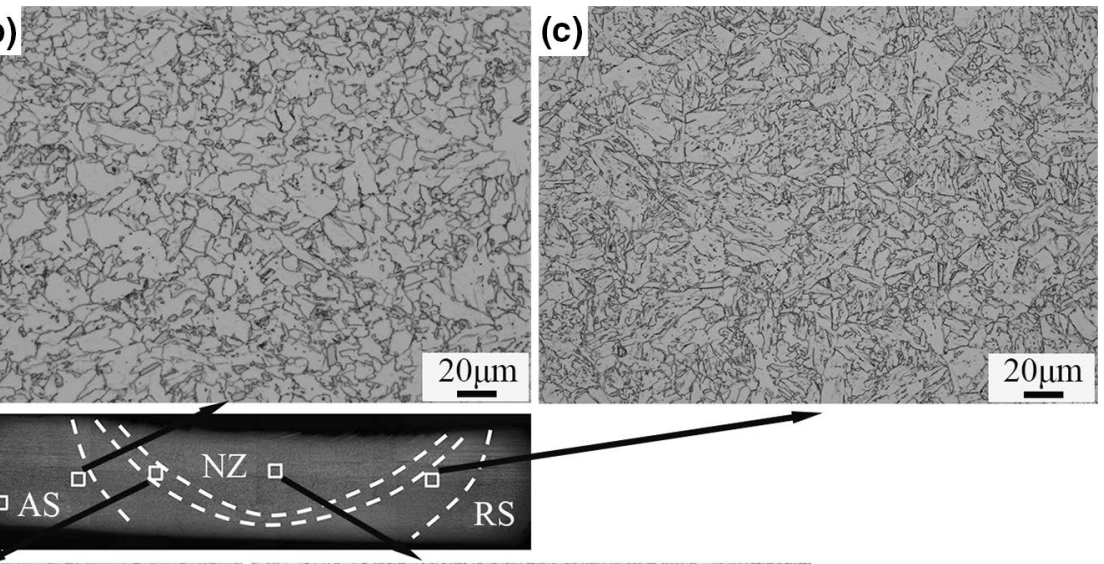

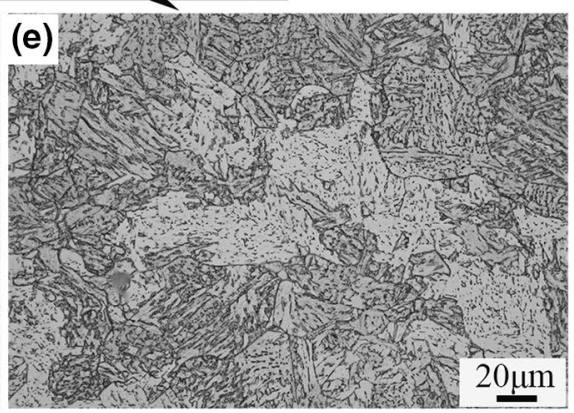

Fig. 4 Microstructures of the welded joint at air cooling: a BM, b OHAZ, c MHAZ, d IHAZ, e NZ 
of ferrite. In this study, the non-uniform microstructure was avoided in the NZ because of the use of W-Re alloy tool.

Figure 5 shows OM images of the OHAZs under various cooling conditions. It was detected that as the cooling medium temperature reduced, the ferritic grains became finer and the microstructure of the OHAZ under liquid $\mathrm{CO}_{2}$ cooling was comparable to that of the original BM. This originated from the short duration time at an elevated temperature in the OHAZ, which decreased the degree of tempering, recovery, and recrystallization. In this case, the OHAZ under liquid $\mathrm{CO}_{2}$ cooling experienced the shortest thermal cycle history, and thus, its microstructure was quite close to that of the BM. By comparison, a rather wide OHAZ consisting of a number of recrystallized ferrites was observed in the OHAZ of FSW X65 by Barnes et al. [13] due to the comparatively high heat input.

The microstructure of the IHAZs under different cooling conditions is shown in Fig. 6, which indicates that the prior austenitic grains were refined remarkably as the cooling medium temperature reduced. Under air cooling, the IHAZ contained GB and LB, while the IHAZ was characterized primarily by $\mathrm{LB}$ and lath martensite (LM) under rapid cooling conditions of water, liquid $\mathrm{CO}_{2}+$ water, and liquid $\mathrm{CO}_{2}$. Because no obvious ferrite phase was discovered, it was extrapolated that the IHAZ was completely austenitized during FSW. Moreover, it was detected that the quantity of the low-temperature transformed products in the IHAZ varied non-monotonically with the cooling medium temperature, and the largest amount of the LM was obtained in the IHAZ under water cooling with middle temperature. However, the microstructural evolution in the IHAZ of FSW pipeline steels did not attract significant attention because in those studies, the IHAZ and NZ exhibited similar microstructural characteristics due to the very high heat input [12-14].

Essentially, as external cooling was introduced and the heat input decreased, the effect of plastic deformation on the microstructure in the NZ became more evident, and thus, different microstructures were produced in the NZ and IHAZ. In this case, there are two competitive factors that influence the phase transformation of the IHAZ under various cooling conditions, mainly relating to the prior austenite size at elevated temperature during FSW and the cooling rate after FSW. With a reduction in the cooling medium temperature, the finer austenite can hinder the quenching tendency and the formation of LB and LM, because many austenitic grain boundaries can interfere the displacive phase transformation process [13]. Nevertheless, a higher cooling rate will facilitate the generation of $\mathrm{LB}$ and $\mathrm{LM}$ owing to the
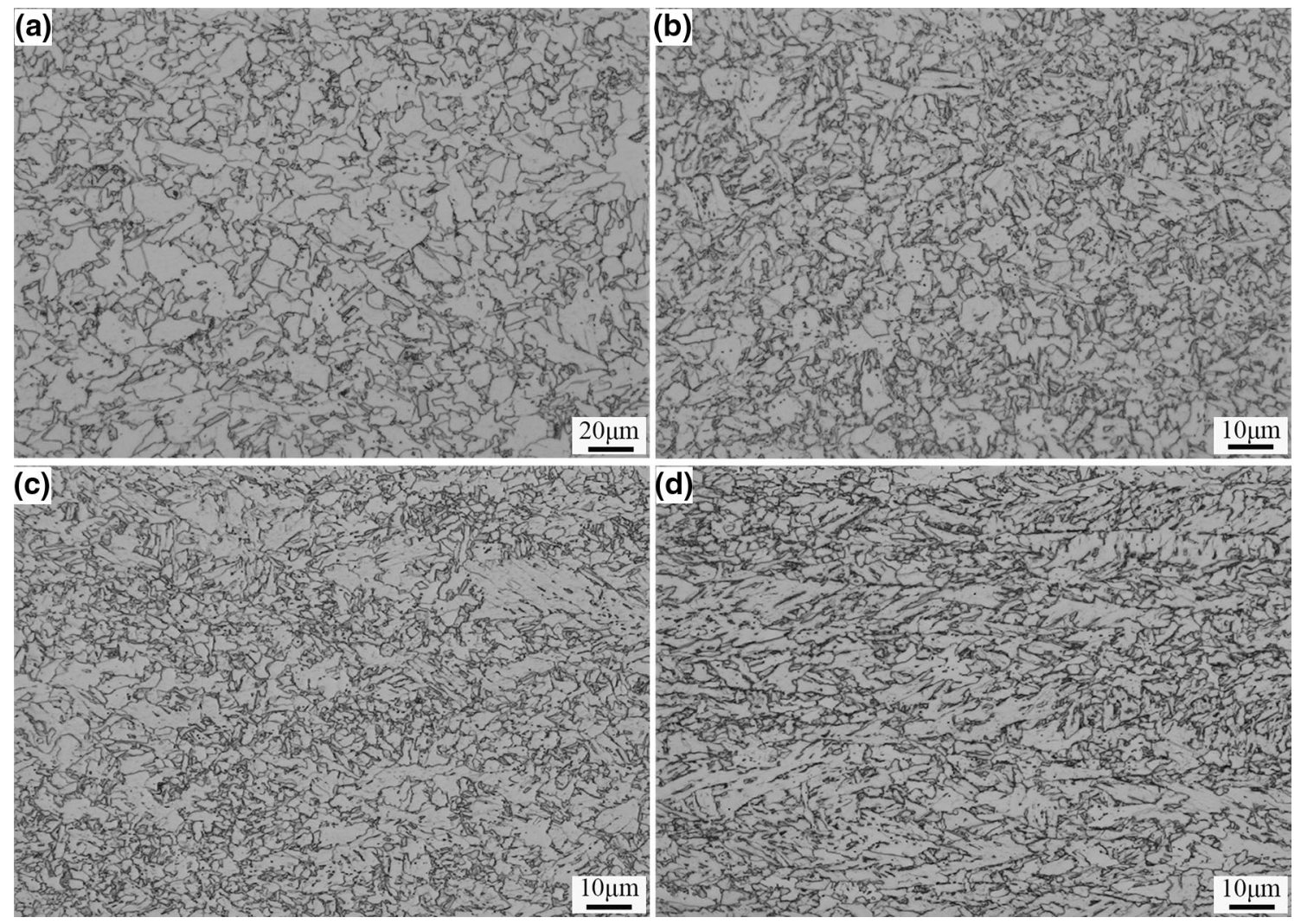

Fig. 5 OM images of the OHAZs at various cooling conditions: a air cooling, b water cooling, $\mathbf{c}$ liquid $\mathrm{CO}_{2}+$ water cooling, $\mathbf{d}$ liquid $\mathrm{CO}_{2}$ cooling 

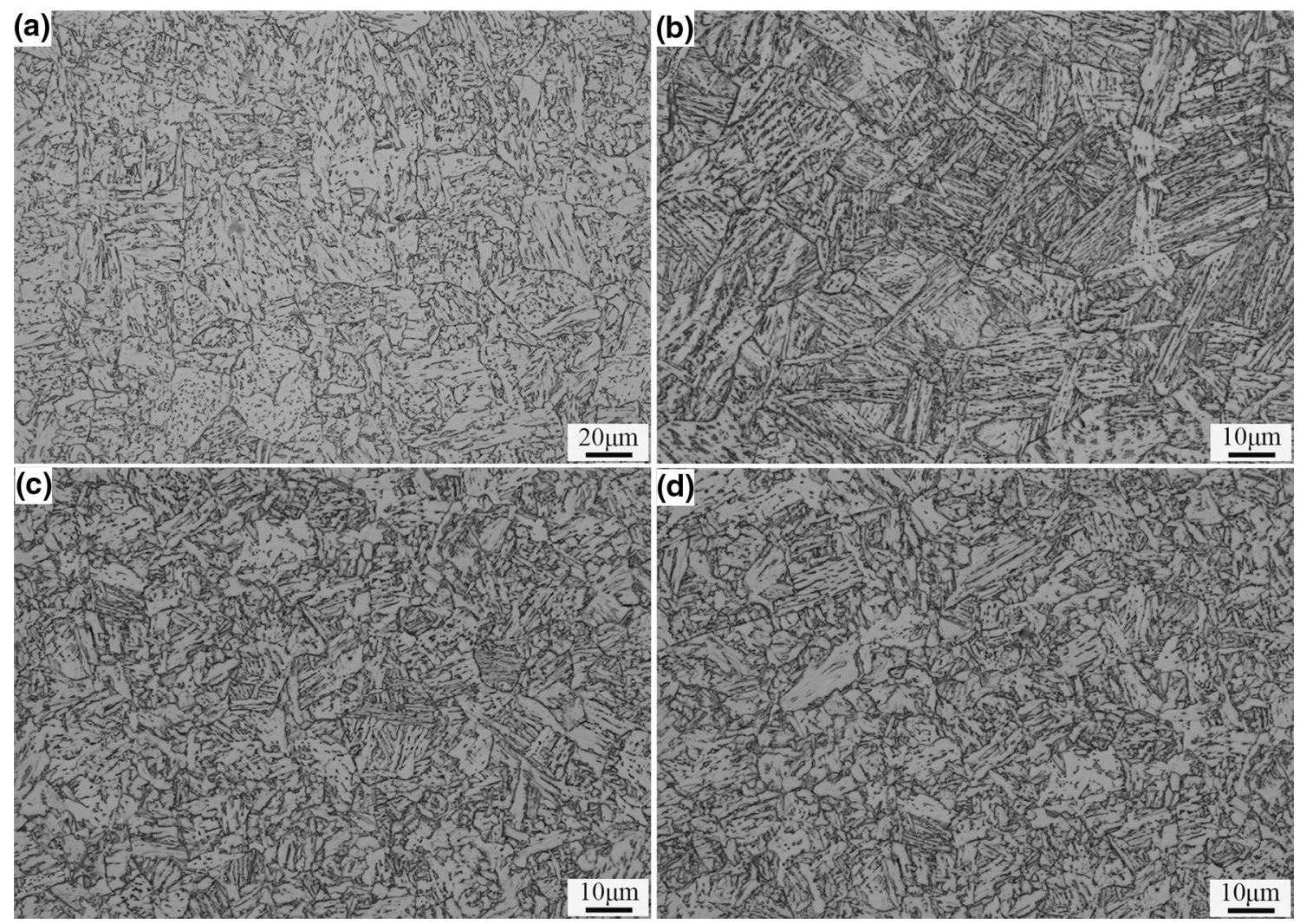

Fig. 6 OM images of the IHAZs under different cooling conditions: a air cooling, b water cooling, $\mathbf{c}$ liquid $\mathrm{CO}_{2}+$ water cooling, $\mathbf{d}$ liquid $\mathrm{CO}_{2}$ cooling

larger undercooling degree [19-21]. Therefore, the largest amount of LM was produced in the IHAZ under water cooling by relying on the integrated action of both the austenitic size and cooling rate.

Figure 7 displays the OM images of the NZs under different cooling conditions. The homogenous microstructure was observed in all the NZs. According to Fig. 7, it was indicated that the prior austenitic grains were refined distinctly with the reduction in cooling medium temperature. This was attributed to the drop in the peak temperature and duration time, thereby effectively retarding the grain growth. Under air cooling, the NZ contained predominantly GB with large M-A constituents and a small amount of coarse LB (Fig. 7a). For the NZ obtained under water cooling, the amount of LB increased obviously, whereas the amount of GB decreased significantly (Fig. 7b). When the liquid $\mathrm{CO}_{2}+$ water cooling was utilized, a large amount of LM emerged and the GB disappeared almost in the NZ (Fig. 7c). Particularly, in the $\mathrm{NZ}$ under liquid $\mathrm{CO}_{2}$ cooling, the amount of LM further enhanced with less existence of LB, but some fine ferrite formed between the LMs (Fig. 7d). Based on the above results, it was suggested that with the drop in cooling medium temperature, a number of low-temperature transformed products were generated depending on the enhanced undercooling. Nevertheless, compared to the case of the IHAZ, the amount of the low-temperature transformed products in the NZ increased monotonically as the cooling medium temperature decreased. In this case, the severe plastic deformation during FSW should be a crucial reason that influences the phase transformation process in the NZ. Although a large amount of LB was obtained in the $\mathrm{NZ}$ of an FSW X80 pipeline steel by decreasing the heat input parameter by Santos et al. [11], LM or the dual phase of LM and ferrite was not observed. This means that there were still a high peak temperature and long duration time, and thus, the heat input during FSW needs to be further decreased to produce an ideal microstructure.

To further investigate the microstructural evolution mechanisms of the NZs under various cooling conditions, TEM images of the NZs are represented in Fig. 8. It can be observed that there was a high density of dislocations in the NZ under air cooling. This should be attributed to the sufficient plastic deformation of austenite at an elevated temperature during FSW under air cooling because of the higher peak temperature and longer duration time. It is well known that a high density of dislocations can retard the coordinated movement of atoms during the austenitic displacive transformation through the mechanical stabilization mechanism and 

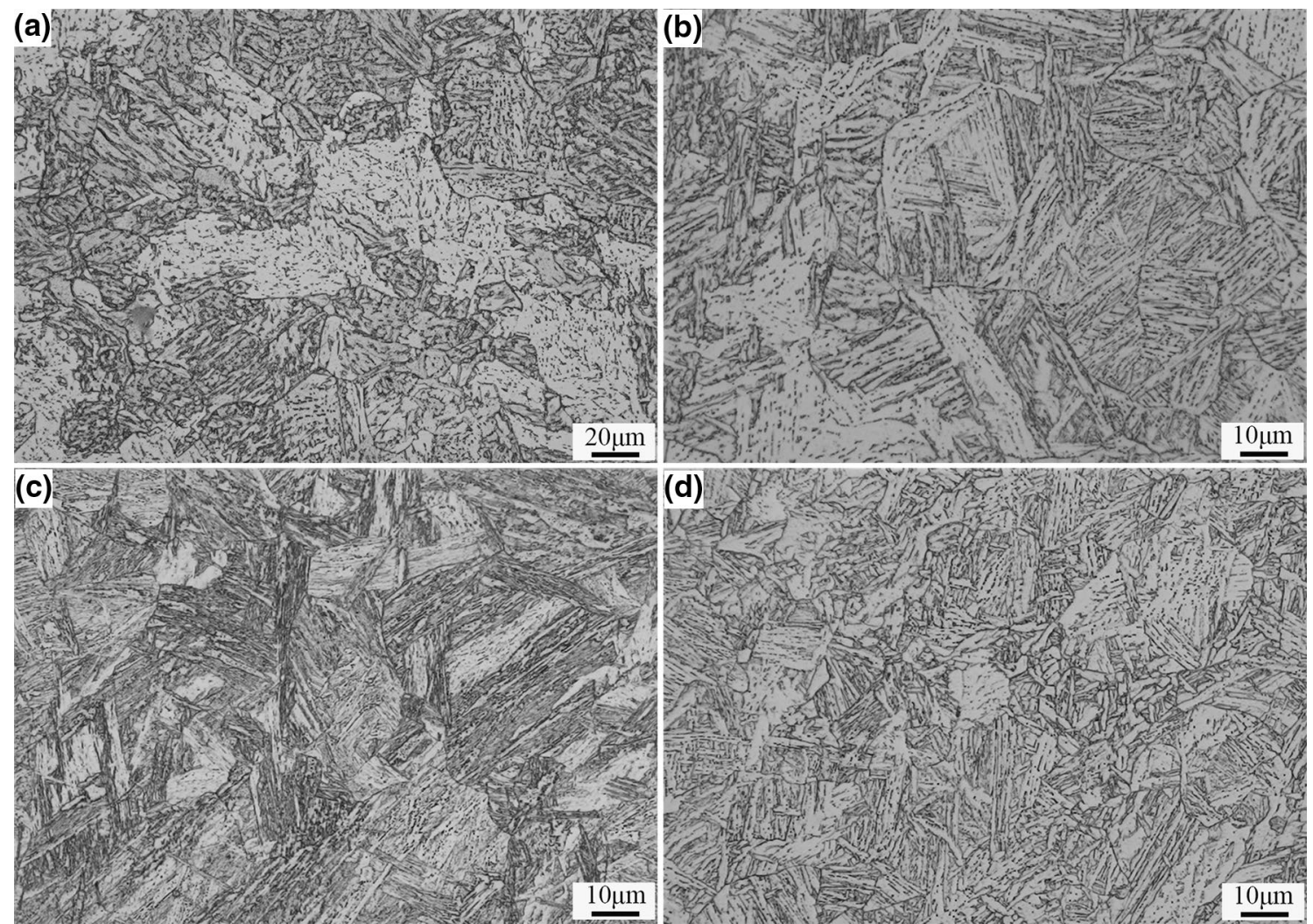

Fig. 7 OM images of the NZs under different cooling conditions: a air cooling, $\mathbf{b}$ water cooling, $\mathbf{c}$ liquid $\mathrm{CO}_{2}+$ water cooling, $\mathbf{d}$ liquid $\mathrm{CO}_{2}$ cooling

facilitate the diffusion of $\mathrm{C}$ at the austenite/ferrite interface via the lower cooling rate and increased number of diffusion paths [22-24]. Therefore, a number of GB and M-A constituents were formed in the NZ under air cooling (Fig. 8a). With the decrease in cooling medium temperature, the insufficient plastic deformation and higher cooling rate caused a displacive phase transformation, producing a higher amount of LB and LM (Fig. 8b-d). Therefore, under the concurrent domination of both cooling rate and plastic deformation, the amount of the low-temperature transformed products in the NZs varied monotonically with the cooling medium temperature.

A certain amount of fine ferrite was found in the NZ under liquid $\mathrm{CO}_{2}$ (Figs. 7d, 8d). Figure 6d shows that the IHAZ under liquid $\mathrm{CO}_{2}$ was austenitized fully because of the absence of ferrite in the IHAZ. By comparison, the NZ having higher peak temperature should also be totally austenitized, and thus, it was deduced that the fine ferrite was not from the austenization process. Furthermore, the peak temperature of the $\mathrm{NZ}$ under liquid $\mathrm{CO}_{2}$ cooling was measured to be $886^{\circ} \mathrm{C}$ by a telemetry system, which was somewhat higher than $A_{c 3}$. Park et al. [25] proposed that the severe plastic deformation at a temperature just higher than $A_{\mathrm{c} 3}$ can create an increase in the transformation temperature from austenite to ferrite. This was the result of the sharply increased nucleation sites at the austenitic matrix and boundaries, thereby leading to the appearance of the deformation-induced ferrite transformation (DIFT) mechanism $[25,26]$. Unlike the PF, the ferrite boundaries obtained through DIFT were not straight, and the fine ferrite contained a high density of dislocations (Fig. 8d). In our previous work, the DIFT phenomenon also was detected in the friction stir spot welded DP780 dual-phase steel, and we proposed that intense deformation can cause tortuous boundaries and a high density of dislocations in ferrite [27]. However, the DIFT can be created only by controlling the peak temperature within a precise range.

Recently, some researchers have studied the microstructural evolution of FSW joints by utilizing external rapid cooling conditions [19-21]. Xue et al. [19, 20] conducted a study on FSW X80 pipeline steel and FSW carbon steel under water cooling, and they found that the NZs were composed of the dual phases of ferrite and bainite, and ferrite and martensite, respectively, which accounted for the peak temperature occurring in the dual-phase region of $\gamma$ and $\alpha$. Additionally, a NZ only consisting of fine ferrite was achieved by using FSW under $\mathrm{CO}_{2}$ cooling by Imam et al. [21], where the peak temperature was less than $A_{\mathrm{c} 1}$. In 

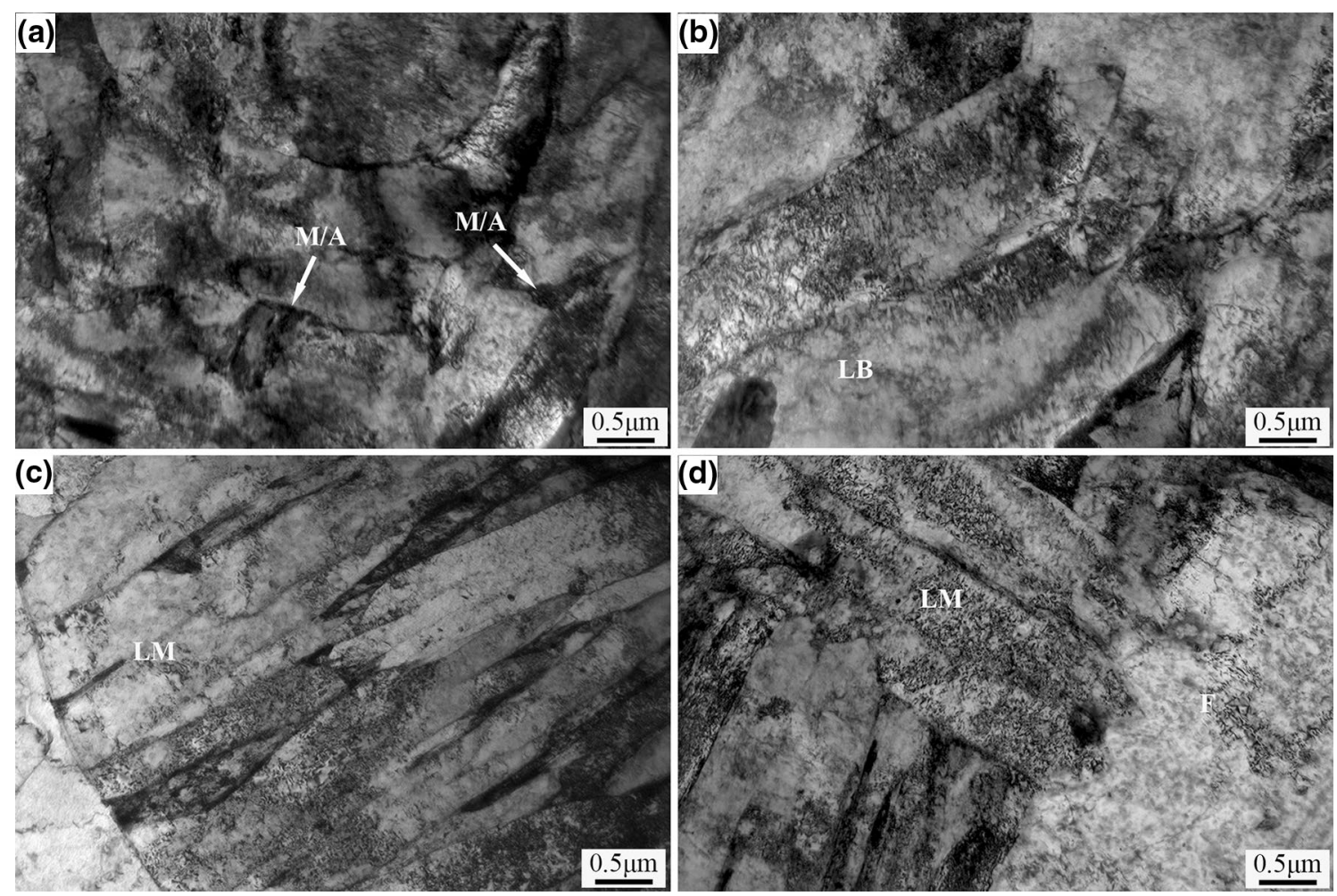

Fig. 8 TEM images in the NZs at various cooling conditions: a air cooling, $\mathbf{b}$ water cooling, $\mathbf{c}$ water + liquid $\mathrm{CO}_{2}$ cooling, $\mathbf{d}$ liquid $\mathrm{CO}_{2}$ cooling

short, relative to the $\mathrm{Al}, \mathrm{Mg}$, and $\mathrm{Cu}$ alloys, the high-strength micro-alloy steels, such as pipeline steels, have a complicated phase transformation during FSW under various cooling conditions $[15,19]$. These competitive factors, including the plastic deformation, degree of austenization, austenitic grain size, and cooling rate, will generate a complex effect on the phase transformation process.

Figures 9 and 10 show the EBSD misorientation and grain boundary distribution maps of the NZs under various cooling conditions, in which the red and black lines represent the high-angle grain boundaries (HAGBs, grain boundary misorientation $\geq 45^{\circ}$ ) and medium-angle grain boundaries (MAGBs, $15^{\circ} \leq$ grain boundary misorientation $<45^{\circ}$ ), respectively. It was pointed out that the HAGBs and MAGBs corresponded to the packet boundaries for the LB and LM, and prior austenite grain boundaries, respectively [28]. The fractions of HAGBs and MAGBs in the NZs under different cooling conditions are presented in Fig. 11. The fraction of HAGBs increased as the cooling medium temperature decreased, which was associated with the fraction of LB and LM, as shown in Fig. 7. Figure 12 shows the average prior austenitic grain sizes of the NZs under various cooling conditions by EBSD, which were 8.2, 4, 3.8, and $3.7 \mu \mathrm{m}$, respectively, under air, water, liquid $\mathrm{CO}_{2}+$ water, and liquid $\mathrm{CO}_{2}$ conditions. Clearly, the grains were refined with the reduction in cooling medium temperature.
Figure 13 reveals the orientation distribution function (ODF) maps of the NZs under different cooling conditions. The shear texture components of D1 ( $\overline{1} \overline{1} 2)[111]$ and D2 $(11 \overline{2})$ [111] were identified in all the NZs, and the texture intensity increased when the cooling medium temperature dropped. Cui et al. [29] suggested that the strong shear texture components were observed within the NZ of FSW austenitic stainless steel, because the NZ was controlled by the shear deformation during FSW. Generally, for the FSW micro-alloy steels, the shear texture component is relatively weaker because of the allotropic phase transformation [30, 31]. However, Abassi et al. [30] pointed out that the shear deformation of FSW X80 steel mainly occurred below the $A_{\mathrm{c} 3}$ temperature, generating a fairly high texture intensity. In this work, for the $\mathrm{NZ}$ under liquid $\mathrm{CO}_{2}$ cooling, the plastic deformation mainly occurred below $A_{\mathrm{c} 3}$ due to the lower peak temperature, and then, it was retained to the environmental temperature through a very rapid cooling rate, eventually producing the strong shear texture shown in Fig. 13d. In contrast, in the NZ under air cooling, the major shear deformation took place above $A_{\mathrm{c} 3}$ and became gradually weak during the slower cooling, obtaining the weak texture shown in Fig. 13a. Therefore, the cooling condition is an important factor that influences the shear texture in the NZs of FSW pipeline steels. Nevertheless, a recent result indicated that a strong shear texture was examined on the 

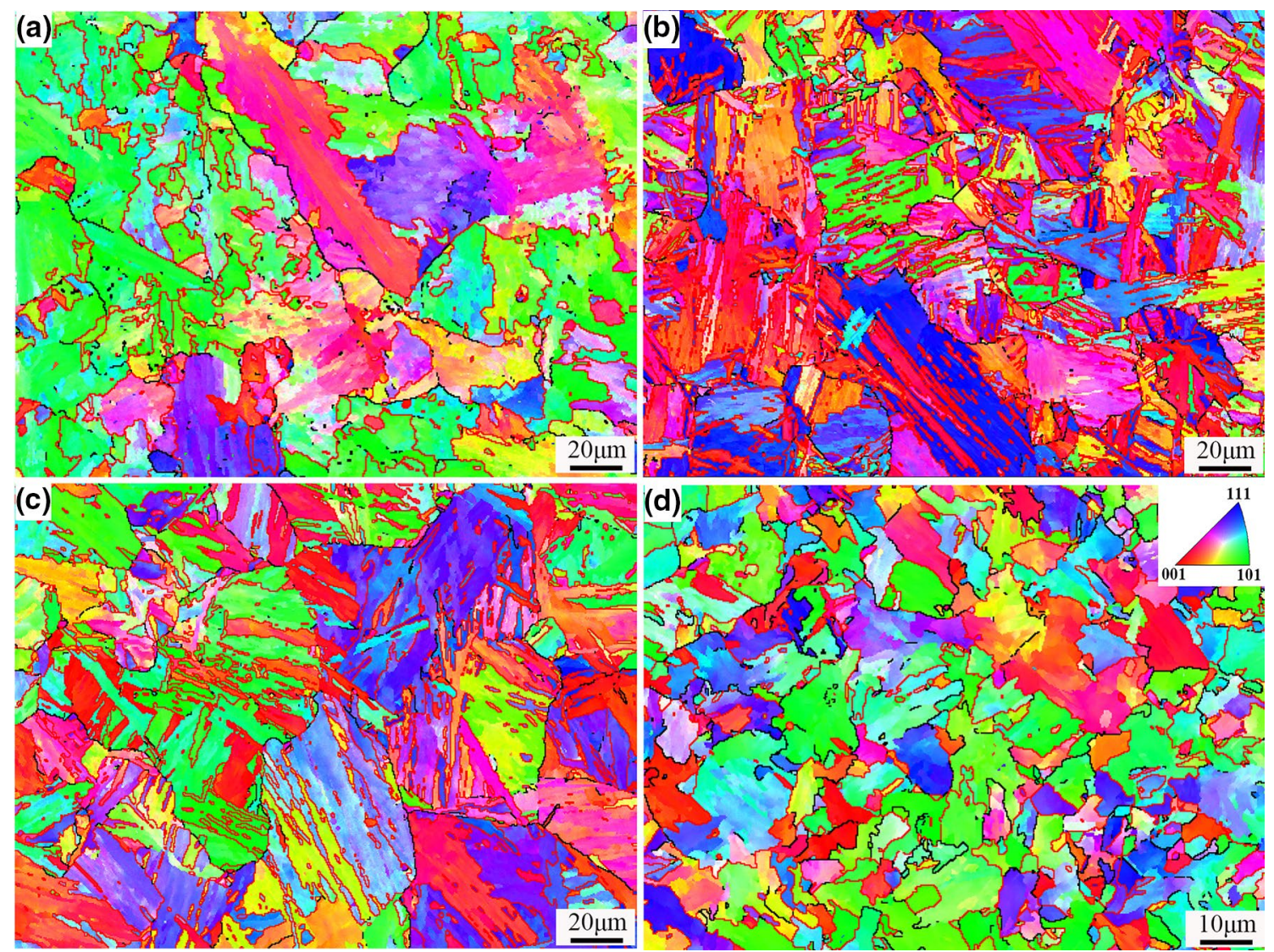

Fig. 9 Misorientation distribution maps in the NZs at various cooling conditions: a air cooling, $\mathbf{b}$ water cooling, $\mathbf{c}$ liquid $\mathrm{CO}_{2}+$ water cooling, $\mathbf{d}$ liquid $\mathrm{CO}_{2}$ cooling

advancing side of the NZ of an FSW X100 steel produced at a higher rotation rate, where the severe plastic deformation at an elevated temperature dominated the texture intensity [16].

\subsection{Effect of the Cooling Conditions on Mechanical Properties of the Joints}

Figure 14 shows the Vickers hardness profiles along the centerline on the cross-sectional joints under various cooling conditions, indicating that the highest hardness existed in the NZ. Under air cooling, the hardness of the IHAZ was the lowest, whereas under rapid cooling conditions of water, water + liquid $\mathrm{CO}_{2}$, and liquid $\mathrm{CO}_{2}$, the lowest hardness occurred in the OHAZ. In this case, the hardness of the FSW joints was governed primarily by the low-temperature transformed products of LB and LM because pipeline steels mainly depend on phase transformation strengthening [7]. As the cooling medium temperature decreased, the hardness of the OHAZ increased continuously because of the mitigation of the tempering softening. For the IHAZ, the highest hardness existed under water cooling given that it contained the largest amount of LB, as shown in Fig. $6 \mathrm{~b}$. The hardness in the NZs increased gradually when the cooling medium temperature reduced, which was closely linked to the amount of the low-temperature transformed products in the NZs (Fig. 7). A hardness difference of $>100 \mathrm{Hv}$ was observed within the NZ of FSW X80 steel by Ozekcin et al. [12], which was the result of the existence of a local hard zone. As mentioned above, this zone strongly relies on the formation of LM through the B element diffusion from the PCBN tool. However, an uniform hardness distribution was detected in this work.

Figure 15 represents the tensile properties of the FSW joints under different cooling conditions. Compared to those of the BM, the FSW joints under various cooling conditions showed a somewhat decreased strength and elongation. Under liquid $\mathrm{CO}_{2}$ cooling, the maximum tensile strength and elongation of the welded joint reached $92 \%$ and $82 \%$ of those of the BM, respectively. Figure 16 shows the failed joints under various cooling conditions. It was observed that the joint under air cooling fractured in the IHAZ, whereas the joints under the other rapid cooling conditions failed in the OHAZ. Because the fracture location of the welded joints is consistent with the lowest hardness zone, the tensile strength and elongation of the FSW joints were those of the lowest 

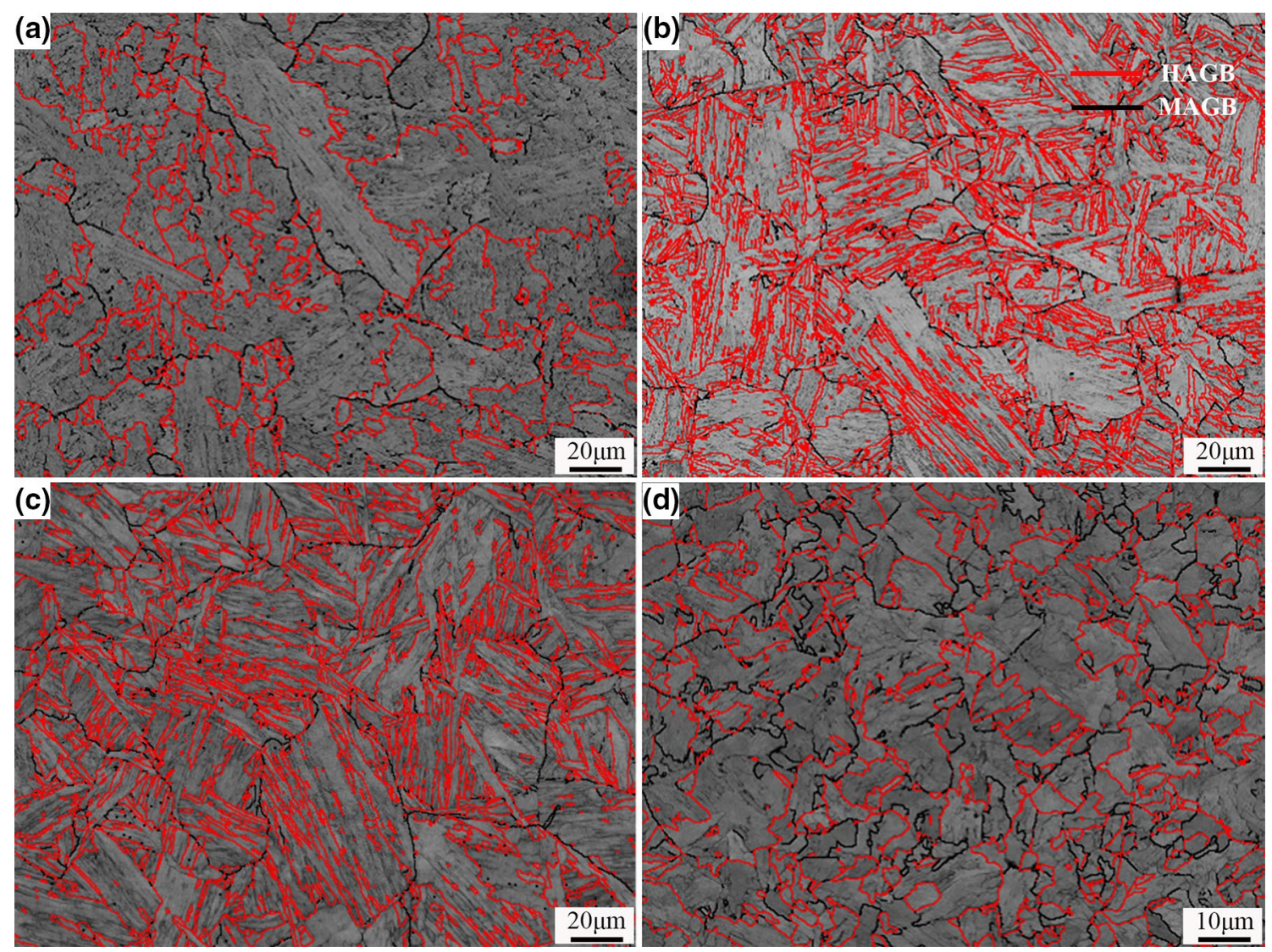

Fig. 10 Grain boundaries distribution maps in the NZs under different cooling conditions: a air cooling, b water cooling, $\mathbf{c}$ liquid $\mathrm{CO}_{2}+$ water cooling, d liquid $\mathrm{CO}_{2}$ cooling

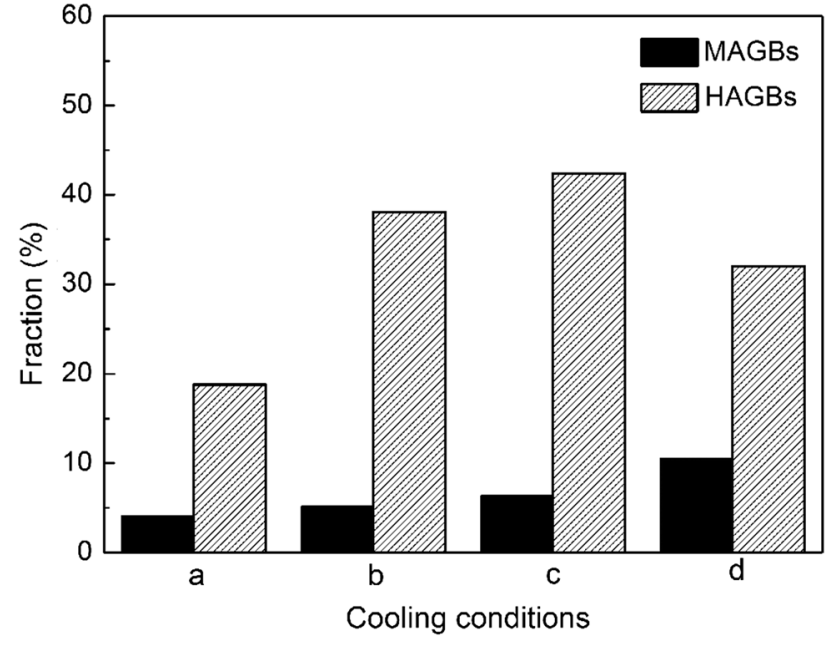

Fig. 11 Fraction of grain boundaries in the NZs at various cooling conditions: a air cooling, b water cooling, $\mathbf{c}$ liquid $\mathrm{CO}_{2}+$ water cooling, d liquid $\mathrm{CO}_{2}$ cooling

hardness zone. Under liquid $\mathrm{CO}_{2}$ cooling, the microstructure in the OHAZ was similar to that of the BM, and therefore, the tensile strength of the FSW joint was comparable to

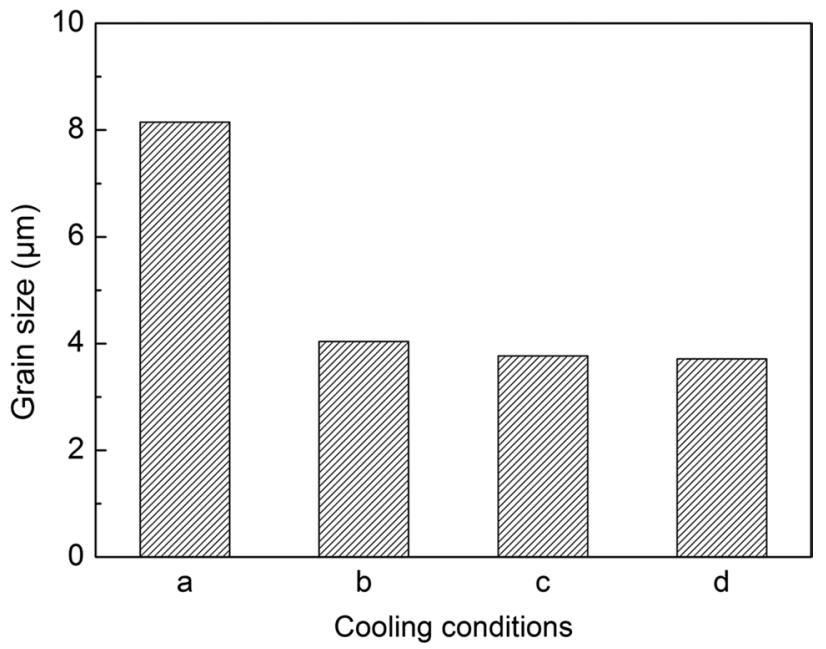

Fig. 12 Average grain sizes of prior austenite in the NZs at various cooling conditions: a air cooling, b water cooling, c liquid $\mathrm{CO}_{2}+$ water cooling, $\mathbf{d}$ liquid $\mathrm{CO}_{2}$ cooling

that of the BM. However, the gage of the tensile specimen covered the NZ, HAZ, and BM, whereas the plastic deformation occurred mainly in the $\mathrm{H}$. Thus, a slightly reduced 


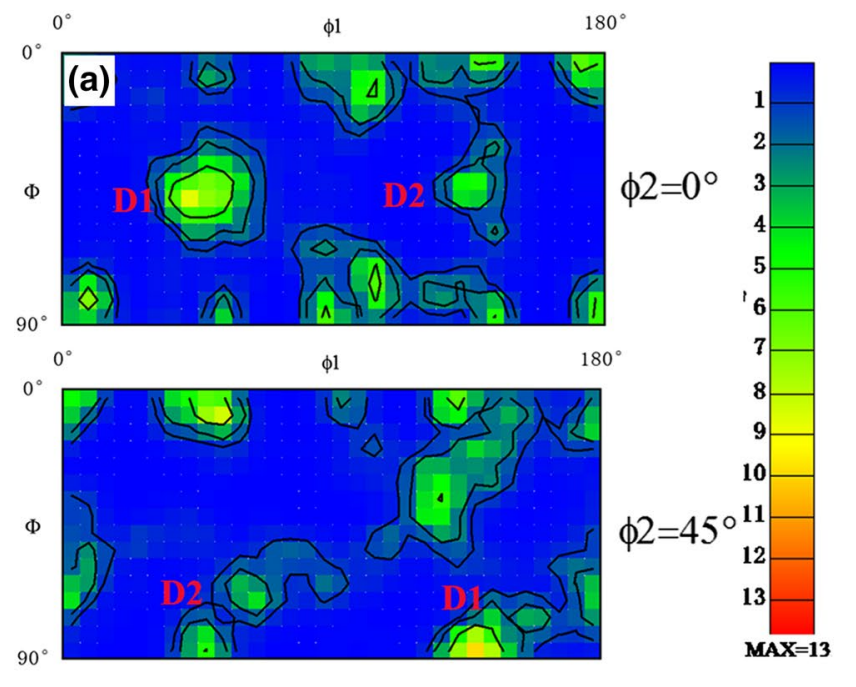

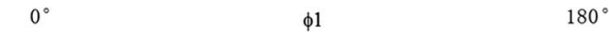
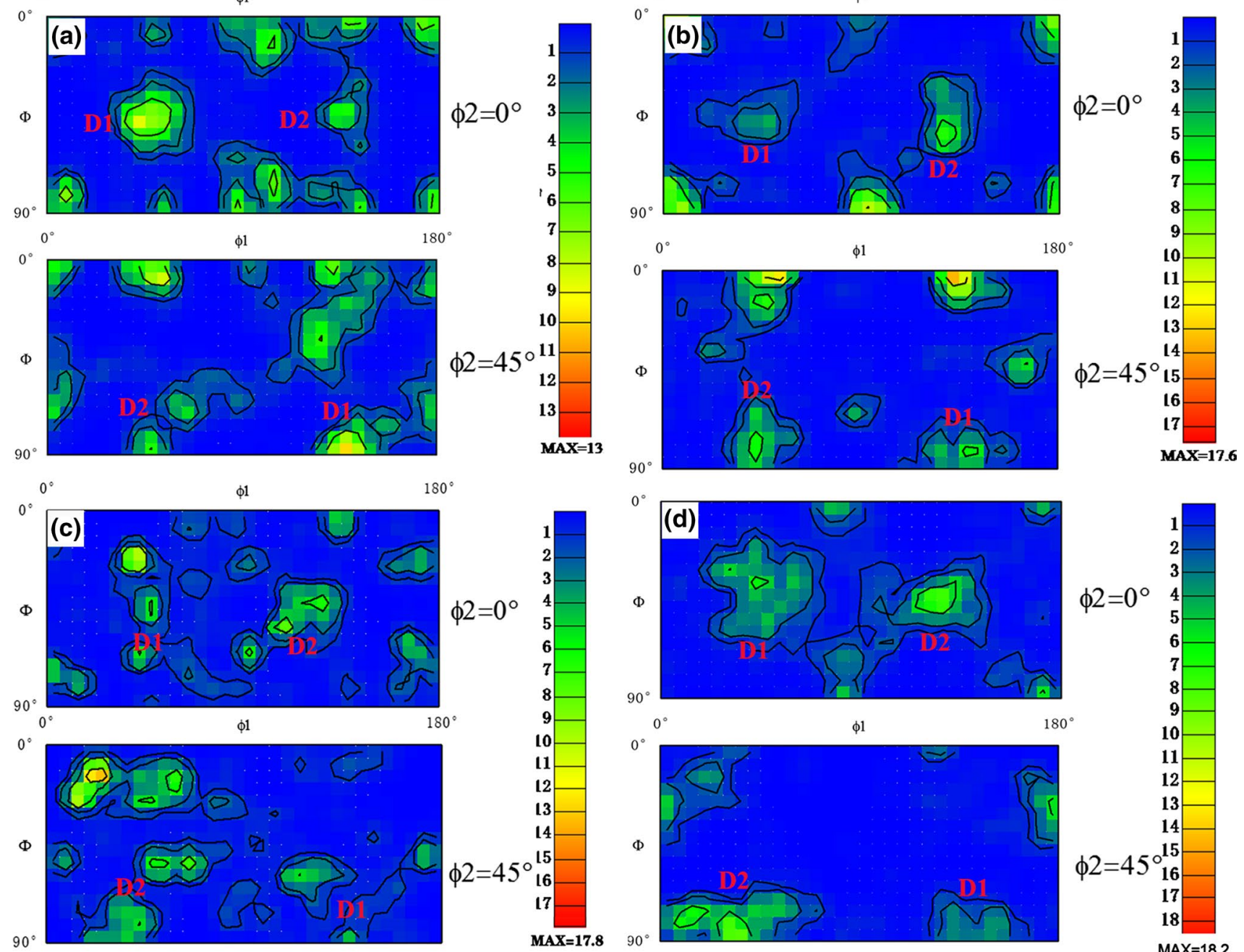

MAX $=17.6$

(e)
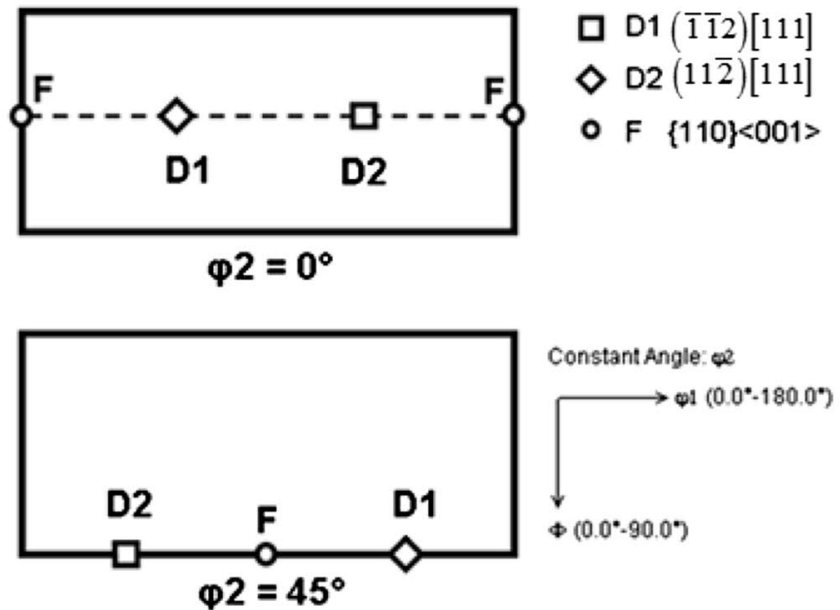

Fig. 13 ODF maps in the NZs under different cooling conditions: a air cooling, b water cooling, $\mathbf{c}$ liquid $\mathrm{CO}_{2}+$ water cooling, $\mathbf{d}$ liquid $\mathrm{CO}_{2}$ cooling, e ideal shear texture of BCC lattice metals 


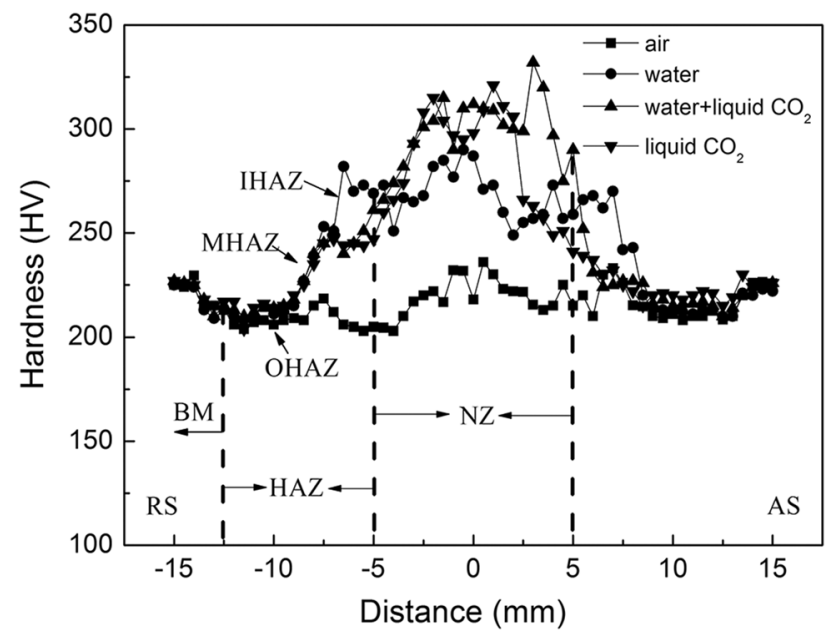

Fig. 14 Hardness profiles of the joints under different cooling conditions

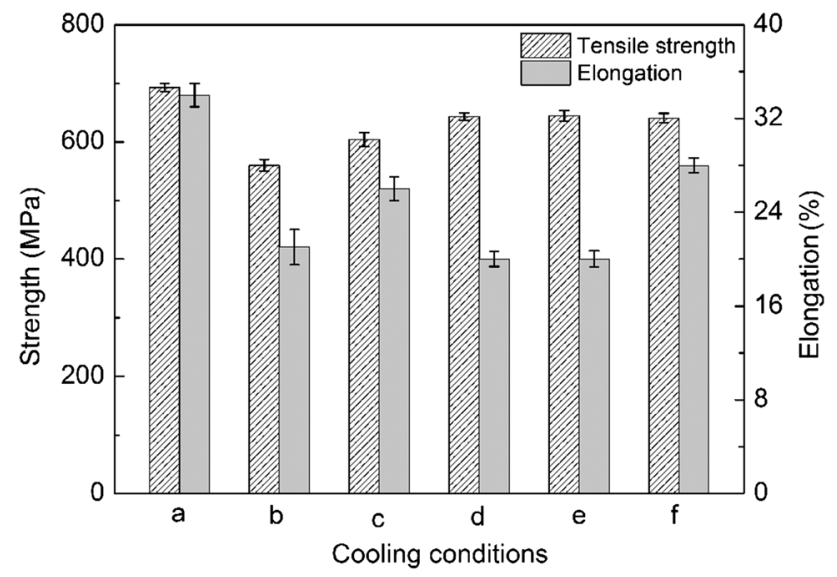

Fig. 15 Tensile properties of BM and joints under different conditions: a BM, b GMAW joint, $\mathbf{c}$ air cooling, $\mathbf{d}$ water cooling, e liquid $\mathrm{CO}_{2}+$ water cooling, f liquid $\mathrm{CO}_{2}$ cooling

elongation was obtained in the FSW joints. By comparison, Midawi et al. [4] conducted tests on GMAW X80 pipeline steels, and they noted that the tensile strength and elongation of the welded joint were up to $80 \%$ and $62 \%$ of those of the BM, respectively. Obviously, compared to those of fusion welded joints, the tensile strength and the elongation of FSW joints under external cooling conditions were improved significantly.

Figure 17 exhibits the impact toughness results of the NZs under different cooling conditions. The impact energy of the NZs under air, water, liquid $\mathrm{CO}_{2}+$ water, and liquid $\mathrm{CO}_{2}$ cooling conditions reached $69 \%, 80 \%, 72 \%$, and $93 \%$ of that of the BM, respectively. The maximum toughness was achieved in the $\mathrm{NZ}$ under liquid $\mathrm{CO}_{2}$, and the lowest toughness was produced in the NZ under air cooling. SEM images of the impact fractural surfaces are shown in Fig. 18. In the (a)

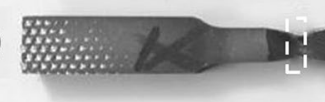

(b)

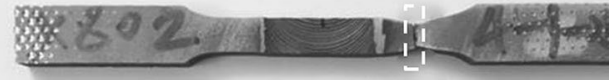

(c)

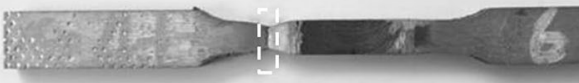

(d)

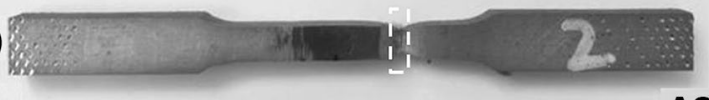

RS AS

Fig. 16 Fracture tensile samples of the joint under different cooling conditions: a air cooling, b water cooling, $\mathbf{c}$ liquid $\mathrm{CO}_{2}+$ water cooling, d liquid $\mathrm{CO}_{2}$ cooling

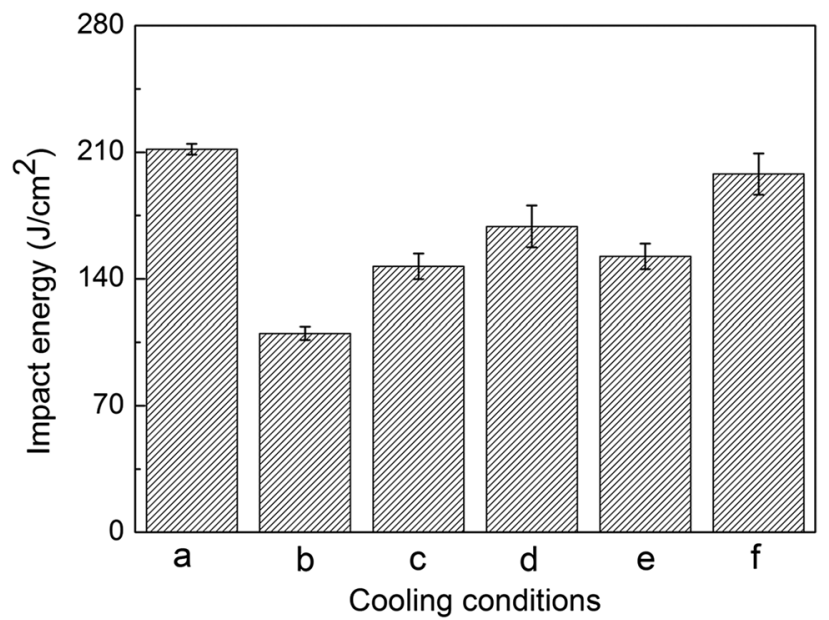

Fig. 17 Impact properties of BM and joints under different conditions: a BM, b GMAW joint, $\mathbf{c}$ air cooling, $\mathbf{d}$ water cooling, e liquid $\mathrm{CO}_{2}+$ water cooling, $\mathbf{f}$ liquid $\mathrm{CO}_{2}$ cooling

comparison, a large number of deep dimples and apparent tearing edges were detected on the fracture surfaces under water cooling and liquid $\mathrm{CO}_{2}$ cooling conditions (Fig. 18b, d), indicating excellent ductile fracture characteristics. Figure 19 shows the crack propagation direction in the NZs under various cooling conditions. The crack propagation direction of the impact sample under air cooling was comparatively straight within the GB (Fig. 19a). Meanwhile, for the NZs under water, liquid $\mathrm{CO}_{2}+$ water, and liquid $\mathrm{CO}_{2}$ cooling conditions, tortuous crack propagation paths were found, as shown in Fig. 19b-d.

Previous reports pointed out that the factors determining the toughness of micro-alloy steels are quite complicated, involving among other the microstructure, grain 

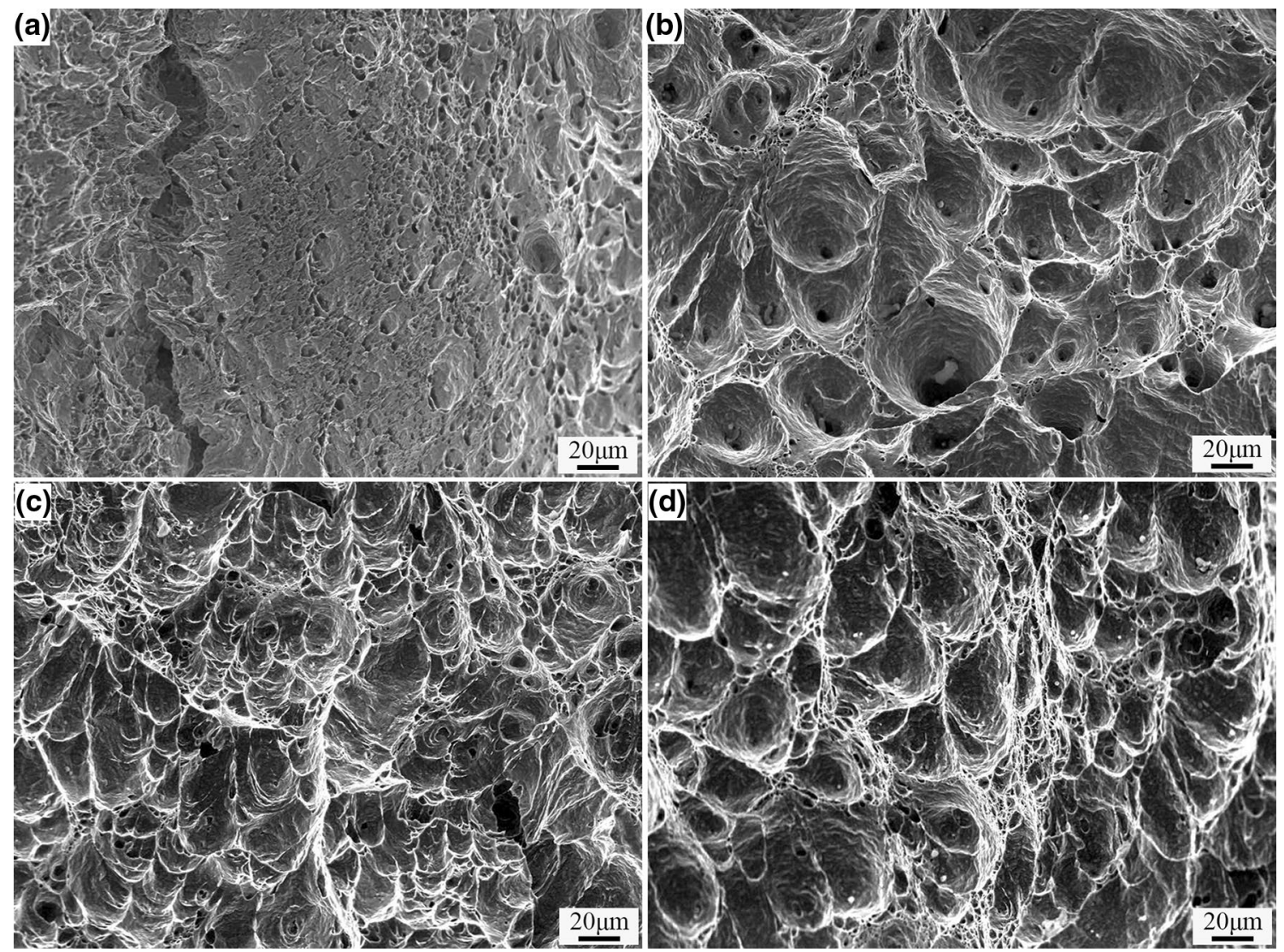

Fig. 18 SEM images of impact fractural surface in the NZ under different cooling conditions: a air cooling, b water cooling, $\mathbf{c}$ liquid $\mathrm{CO}_{2}+$ water cooling, $\mathbf{d}$ liquid $\mathrm{CO}_{2}$ cooling

size, and size and fraction of the second phase [15, 32]. It was demonstrated that the bainite packet as a strong obstacle can arrest effectively the crack propagation and improve notably the toughness because of the presence of numerous HAGBs [33]. For the NZ under water cooling, the direction of the crack propagation path was changed when the crack encountered another bainite packet (Fig. 19b). Moreover, the crack propagation paths were tortuous and more energy was consumed during the crack propagation, finally improving the toughness of joints. However, the crack propagation direction for the NZ under air cooling was across the entire GB. Kang et al. [34] proposed that GB offered the lower resistance to the crack propagation owing to the absence of bainite packets. Furthermore, the coarse M-A constituents often were potential crack nucleation locations, which are harmful to the impact toughness $[33,35]$. As a result, the NZ under air cooling exhibited an inferior toughness relative to the NZ under water cooling. Compared to the case of the LB, there is a significant stress concentration between the supersaturated martensite laths, which can promote the crack propagation [36]. Therefore, the $\mathrm{NZ}$ under liquid $\mathrm{CO}_{2}+$ water cooling exhibited reduced toughness, although it contained a higher fraction of HAGBs compared to that of the NZ under water cooling.

As we know, when the soft second phase of ferrite or retained austenite is introduced into the hard phase of martensite, the strong stress concentration within martensite could be relaxed by self-adjustment between the hard and soft phases, finally improving the toughness [36, 37]. Xue et al. [20] reported that the fine ferrite phase in the dual phase of ferrite and martensite could restrain the formation of cleavage crack and change the crack propagation direction. In Fig. 19d, it can be observed that the crack propagation was arrested by the fine ferrite obtained by the DIFT. Therefore, an excellent toughness was achieved in the NZ under liquid $\mathrm{CO}_{2}$ cooling.

Midawi et al. [5] indicated that the impact toughness in the HAZ of GMAW X80 pipeline steel joints only reached $52 \%$ of that of the BM, which was far lower than that of the NZ by FSW under external rapid cooling conditions. The external rapid cooling is an effective way to improve the strength, plasticity, and toughness of FSW high-strength pipeline steel joints. Besides, some studies reported that the FSW at the strictly low peak temperature of $<A_{\mathrm{c} 1}$, namely the ferrite phase field, caused easily poor plastic flow and 

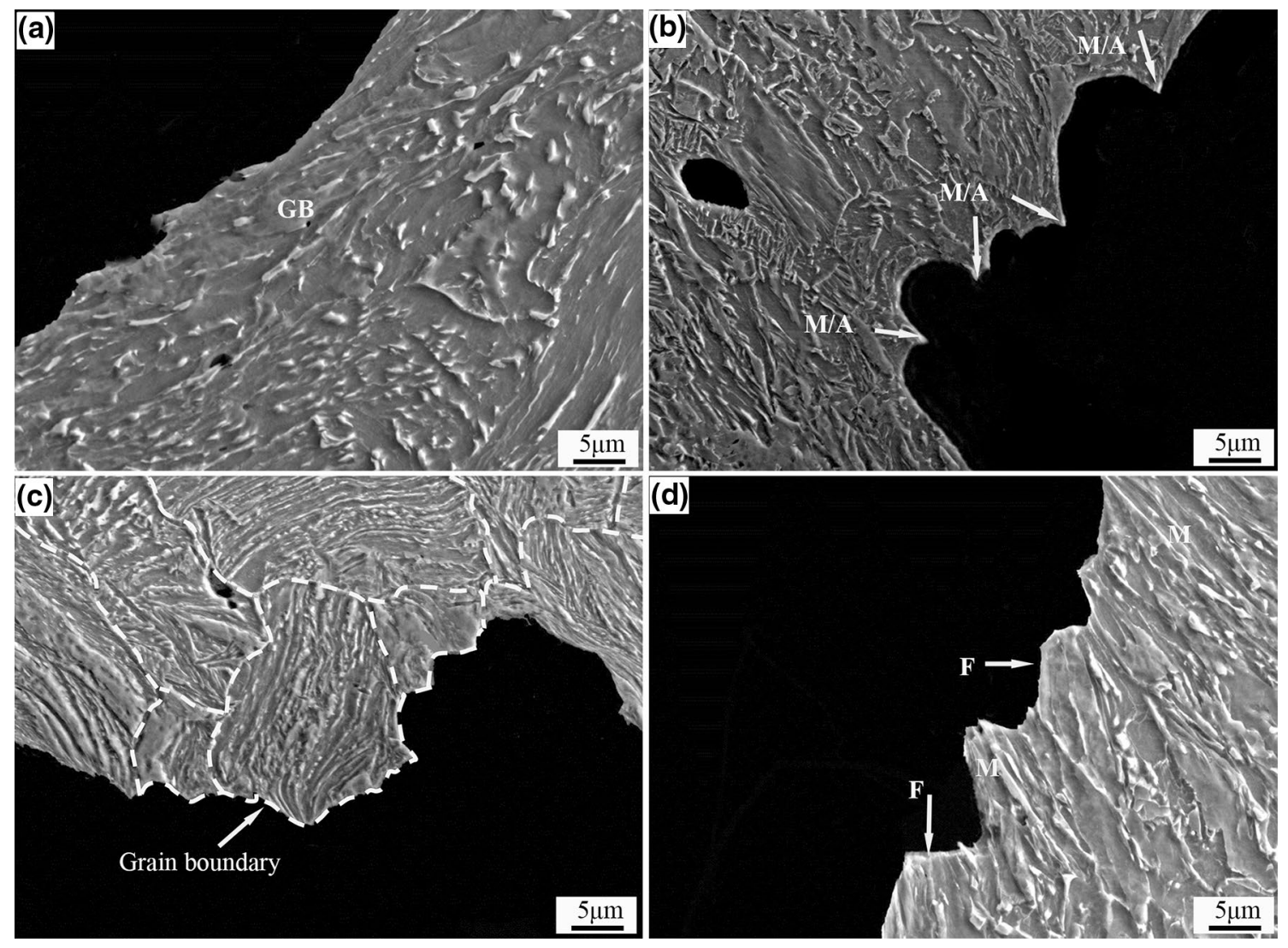

Fig. 19 SEM images of impact crack propagation paths under different cooling conditions: a air cooling, $\mathbf{b}$ water cooling, $\mathbf{c}$ liquid $\mathrm{CO}_{2}+$ water cooling, d liquid $\mathrm{CO}_{2}$ cooling

a great deformation force on the FSW tool, so that the tool was destroyed readily $[38,39]$. Therefore, it is important to select a suitable cooling condition.

\section{Conclusion}

Defect-free joints were obtained under different cooling conditions. Coarse GB was observed in the NZ under air cooling, whereas LB and LM were detected in the NZs under rapid cooling conditions, and some fine ferrite appeared in the $\mathrm{NZ}$ under liquid $\mathrm{CO}_{2}$ cooling besides the LM. The shear texture components of D1 (1̄12)[111] and D2 $(11 \overline{2})[111]$ were identified in the NZs, and the texture intensity increased after utilizing the rapid cooling conditions. Under various cooling conditions, the highest hardness was in the NZ. Under air cooling, the lowest hardness occurred in the IHAZ, while under other rapid cooling conditions, the OHAZ had the lowest hardness. Under the rapid cooling conditions of water, liquid $\mathrm{CO}_{2}+$ water, and liquid $\mathrm{CO}_{2}$, the tensile strength of the joints reached $92 \%$ of that of the $\mathrm{BM}$, and the fracture occurred in the OHAZ. The maximum impact toughness was obtained in the $\mathrm{NZ}$ under liquid $\mathrm{CO}_{2}$ cooling, which was up to $93 \%$ of that of the BM. Therefore, rapid cooling is beneficial to improve the strength, plasticity, and toughness of FSW pipeline steel joints.

Acknowledgements This work was financially supported by the National Nature Science Foundation of China (Nos. 51774085 and 51671190), the Fundamental Research for the Chinese Central Universities (No. N170704013), and the National Key Research and Development Program of China (No. 2017YFB0305004).

\section{References}

[1] H.K. Sung, S.Y. Shin, W. Cha, K. Oh, S. Lee, N.J. Kim, Mater. Sci. Eng. A 528, 3350 (2011)

[2] S.Y. Shin, G. Gong, S. Kim, S. Lee, Metall. Mater. Trans. A 38, $1012(2007)$

[3] C. Zhang, Y.F. Cheng, J. Mater. Eng. Perform. 19, 834 (2010)

[4] A.R.H. Midawi, C.H.M. Simha, A.P. Gerlich, Int. J. Press. Vessels Pip. 168, 258 (2018)

[5] A.R.H. Midawi, E.B.F. Santos, N. Huda, A.K. Sinha, R. Lazor, A.P. Gerlich, J. Mater. Process. Technol. 226, 272 (2015)

[6] J.A. Avila, E. Lucon, J. Sowards, P.R. Mei, A.J. Ramirez, Metall. Mater. Trans. A 47, 2855 (2016)

[7] H. Aydin, T.W. Nelson, Mater. Sci. Eng. A 586, 313 (2013) 
[8] H.S. Shin, K.T. Park, C.H. Lee, K.H. Chang, Ksce J. Civ. Eng. 19, $1431(2015)$

[9] A. Elrefaey, Y. Javadi, J.A. Francis, M.D. Callaghan, A.J. Leonard, Int. J. Press. Vessels Pip. 165, 20 (2018)

[10] R.S. Mishra, Z.Y. Ma, Mater. Sci. Eng. R 50, 1 (2005)

[11] T.F.A. Santos, T.F.C. Hermenegildo, C.R.M. Afonso, R.R. Marinho, M.T.P. Paes, A.J. Ramirez, Eng. Fract. Mech. 77, 2937 (2010)

[12] A. Ozekcin, H.W. Jin, J.Y. Koo, N.V. Bangaru, R. Ayer, G. Vaughn, R. Steel, S. Packer, Int. J. Offshore Polar Eng. 14, 284 (2004)

[13] S.J. Barnes, A.R. Bhatti, A. Steuwer, R. Johnson, Metall. Mater. Trans. A 43, 2342 (2012)

[14] A. Tribe, T.W. Nelson, Eng. Fract. Mech. 150, 58 (2015)

[15] G.M. Xie, H.B. Cui, Z.A. Luo, R.D.K. Misra, G.D. Wang, Mater. Sci. Eng. A 704, 401 (2017)

[16] H.B. Cui, G.M. Xie, Z.A. Luo, J. Ma, G.D. Wang, R.D.K. Misra, J. Alloys Compd. 681, 426 (2016)

[17] H.J. Zhang, H.J. Liu, Mater. Des. 45, 206 (2013)

[18] P. Xue, B.L. Xiao, Q. Zhang, Z.Y. Ma, Scr. Mater. 64, 1051 (2011)

[19] P. Xue, H. Zhao, Y. Komizo, Z.Y. Ma, in Proceedings of the 1st International Joint Symposium on Joining and Welding, Osaka, Japan, November 6-8 (2013)

[20] P. Xue, B.L. Xiao, W.G. Wang, Q. Zhang, D. Wang, Q.Z. Wang, Z.Y. Ma, Mater. Sci. Eng. A 575, 30 (2013)

[21] M. Imam, R. Ueji, H. Fujii, J. Mater. Process. Technol. 230, 62 (2016)

[22] H.K.D.H. Bhadeshia, Mater. Sci. Eng. A 273-275, 58 (1999)

[23] P.H. Shipway, H.K.D.H. Bhadeshia, Mater. Sci. Technol. 11, 1116 (1995)

[24] S.H.C. Park, Y.S. Sato, H. Kokawa, K. Okamoto, S. Hirano, M. Inagaki, Metall. Mater. Trans. A 40, 625 (2009)
[25] J.K. Park, K.H. Kim, J.H. Chung, S.Y. Ok, Metall. Mater. Trans. A 39, 235 (2008)

[26] P.D. Hodgson, M.R. Hickson, R.K. Gibbs, Scr. Mater. 40, 1179 (1999)

[27] G.M. Xie, H.B. Cui, Z.A. Lou, W. Yu, J. Ma, G.D. Wang, J. Mater. Sci. Technol. 32, 326 (2016)

[28] S.H.C. Park, Y.S. Sato, H. Kokawa, K. Okamoto, S. Hirano, M. Inagaki, Scr. Mater. 49, 1175 (2003)

[29] H.B. Cui, G.M. Xie, Z.A. Lou, J. Ma, G.D. Wang, R.D.K. Misra, Mater. Des. 106, 463 (2016)

[30] M. Abbasi, T.W. Nelson, C.D. Sorensen, Metall. Mater. Trans. A 43, 4940 (2012)

[31] F.C. Liu, Y. Hovanski, M.P. Miles, C.D. Sorensen, T.W. Nelson, J. Mater. Sci. Technol. 34, 39 (2018)

[32] H.F. Lan, L.X. Du, R.D.K. Misra, Mater. Sci. Eng. A 611, 194 (2014)

[33] Y. You, C.J. Shang, W.J. Nie, S. Subramanian, Mater. Sci. Eng. A 558, 692 (2012)

[34] J.S. Kang, J.B. Seol, C.G. Park, Mater. Charact. 79, 110 (2013)

[35] Y.L. Zhou, T. Jia, X.J. Zhang, Z.Y. Liu, R.D.K. Misra, Mater. Sci. Eng. A 626, 352 (2015)

[36] M. Calcagnotto, Y. Adachi, D. Ponge, D. Raabe, Acta Mater. 59, $658(2011)$

[37] J.P. Wang, Z.G. Yang, B.Z. Bai, H.S. Fang, Mater. Sci. Eng. A 369, 112 (2004)

[38] H.K.D.H. Bhadeshia, T. DebRoy, Sci. Technol. Weld. Join. 14, 193 (2009)

[39] R. Dimitriu, H.K.D.H. Bhadeshia, Mater. Sci. Technol. 23, 1127 (2007) 